\title{
Effects of Power Allocation and User Mobility on Non-Orthogonal Multiple Access Using Successive Interference Cancellation
}

\author{
Khoirun Ni'amah $^{1 *}$, Solichah Larasati ${ }^{2}$ and Raudhatul Jannah ${ }^{3)}$ \\ ${ }^{1,2,3)}$ Faculty of Telecommunication and Electrical Engineering, Institut Teknologi Telkom Purwokerto, Indonesia \\ Corresponding Email: *) irun@ittelkom-pwt.ac.id
}

\begin{abstract}
This research based on simulation to show impact of the power allocation on Non-Orthogonal Multiple Access (NOMA) using Successive Interference Cancellation (SIC). NOMA used superposition code (SC) on the transmitter and SIC on the receiver. NOMA has two categories power domain (PD) and code domain (CD). This research based on PD-NOMA simulated for downlink. The number of users who use the same recourse block are divided into two conditions: user with apply SIC and without SIC base on the value of channel gain from each user. Applying SC on the transmitter and SIC on the receiver will cancel of interference. Novelties of this research are the best performance of power allocation and user mobility based on parameter BER and SNR. Allocation of the power transmit based on value of channel gain every user, where user with value of channel gain is low will be allocated high power transmit, and otherwise. The best result performance of BER vs SNR used ratio power transmit $0.45 \mathrm{~dB}: 0.55 \mathrm{~dB}$, BER $10^{-5}$ get value $S N R$ for $U_{1}=17 \mathrm{~dB}$ and $U_{2}=18 \mathrm{~dB}$. The best performance SNR for mobility of user with speed $U_{1}, U_{2}=40 \mathrm{~km} / \mathrm{h}$ value SNR $18 \mathrm{~dB}$ for BER $10^{-5}$. This research has proposed to show impact of power transmit and interference in performance NOMA.
\end{abstract}

Keywords: Non-Orthogonal Multiple Access (NOMA), Successive Interference Cancellation (SIC), and Power Domain (PD).

\section{INTRODUCTION}

The increasing demand for cellular services requires a new radio access technology concept to fulfill service needs without ignore services quality. In recent years the Third Generation Partnership Project (3GPP) initiated a discussion on further steps in the evolution $4^{\text {th }}$ generation namely Long-Term Evolution (LTE) release 12 towards future technologies. In addition, some of the initial discussions on the $5^{\text {th }}$ namely fifth generation (5G)[1].

The vision of the $5 \mathrm{G}$ cellular communication system not only focus on enhanced Mobile Broadband (eMBB) and Ultra Reliable Low Latency Communication (URLLC). 5G also has a vision of Massive Machine Type Communication (mMTC). This is due to the increase in traffic demand in 2020 [2]. Using multiple access techniques that are more suitable, namely effective and efficient use of bandwidth from the previous generation, is one solution to fulfill these challenges.

The Orthogonal Multiple Access (OMA) scheme is applied to the $4 \mathrm{G}$ network. The $4 \mathrm{G}$ network uses OFDMA multiple access techniques for downlink transmission and Single Carrier Frequency Multiple Access (SC-FDMA) for uplink transmission. The Non-Orthogonal Multiple Access (NOMA) scheme is a candidate for multiple access techniques that will be applied to $5 \mathrm{G}$ technology [3] [4]. This scheme can be used to increase the number of devices that can be connected in area when compared to Orthogonal Multiple Access (OMA). NOMA as an evolution of the Orthogonal Frequency Division Multiple Access (OFDMA) which was previously applied to $4 \mathrm{G}$ allows multi users to access the frequency channel at the same time non-orthogonally based on the power domain or code [3],[5]. NOMA allows a set of subcarriers to be used by more than one user with different sending power levels. The number of users using the same subcarrier is limited to control the complexity of signal detection at the receiver.

Provisions for using the transmit power level for each user are considered based on the multiuser transmit power allocation method used. In addition, the condition of the channel used also affects the amount of transmit power to be used. On the receiver side, a multiuser detection simulation is performed using Successive Interference Cancellation (SIC). SIC is used to eliminate interference between multi-users who use the same time frequency resource. SIC is only applied to users with a small power allocation.

Base on research [6] researching multiple access NOMA as a promising new power domain for future radio access services by implementing SIC to minimize interference on the receiver side so that it has a better tradeoff than OMA which is widely used in $4 \mathrm{G}$ communication systems. The results of this study indicate the level of profit in increasing the throughput value of NOMA to OMA. The results in [7] showed that the channel capacity in NOMA is greater than that of the OMA channel.

Base on research [12] discussed an optimization scenario in the literature when PD-NOMA is combined with one or more of the candidate schemes and technologies for 5G. NOMA-supported transmission schemes are increasingly being seen as promising dual access schemes for the future wireless networks. This NOMA scheme is very suitable for future networks, so this study uses the NOMA scheme to see the effect of power allocation and user mobility. 
Simulation performance in this study using the Matlab R2019a software by calculating the value of Signal to Noise Ratio (SNR) and Bit Error Rate (BER). This research simulates the Power Domain Non-Orthogonal Multiple Access (PD-NOMA) by applying successive interference cancellation (SIC) on the receiver side. Performance analysis is show parameters in the form of Signal to Noise Ratio (SNR) and Bit Error Rate (BER) based on the comparison of the transmit power and user mobility.

Structure of this research are devided in five sections. The first section discusses the background of research, especially in cellular technology. The second section will discuss the access methods of NOMA, PD-NOMA and SIC which are used as a basis for analyzing the effect of power allocation on NOMA. In the fourth section, we will discuss the analysis and simulation results of the effect of power allocation on NOMA. Conclusions and future work will be presented in the fifth section of this paper.

\section{RESEARCH METHOD}

\section{A. Non-Orthogonal Multiple Access (NOMA)}

In conventional OMA schemes, multi-users are allocated with orthogonal radio resources in time, frequency, or code domains. In theory, there is no interference between users because the resource allocation is orthogonal, so simple user detection can be used to separate signals from different users [8]. However, in the OMA scheme, the maximum number of users that can be supported is limited by orthogonal resource scheduling [9]. The solution to solve problem in OMA is to use the NOMA technique, where one subcarrier can be used by more than one user with different transmitting power levels. The conditions for using the transmit power level for each user are considered based on the multiuser transmit power allocation method used. In addition, the condition of the channel used also affects the transmission power level to be used.

There are two types of NOMA, namely power domain and code domain. In this research using the type of NOMA with the power domain. The NOMA scheme with the power domain can be applied on the downlink and uplink side. In the PD-NOMA system, the subcarrier can be used by multiple users simultaneously by applying the superposition code (SC) on the BS, and the user is applied SIC to remove signals from other users.

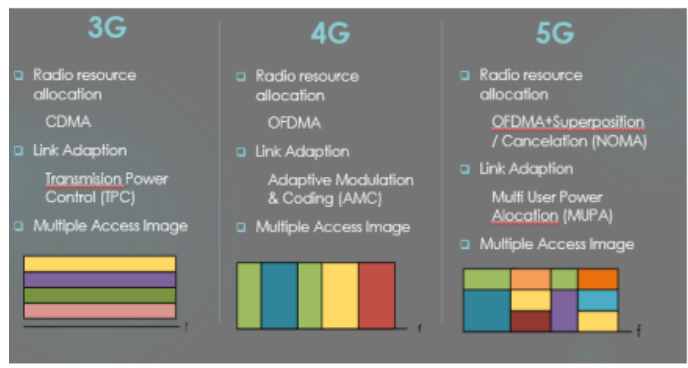

Figure 1. Parameter different of $3 \mathrm{G}, 4 \mathrm{G}$ and 5G [3]

Figure 1 show the technical differences between $3 \mathrm{G}$, $4 \mathrm{G}$, and $5 \mathrm{G}$. The signal wave equation used in $5 \mathrm{G}$ is predicted to adopt OFDM (DFT-s-OFDM) like the signal waves previously used in $4 \mathrm{G}$. Based on the parameter table in Figure 1, it can be seen that power allocation is an important part of this NOMA scheme. This is one of the main factors that different the NOMA and OFDMA used in $4 \mathrm{G}$ dan $5 \mathrm{G}$.

\section{B. Basic Concept PD-NOMA}

NOMA scheme with the power domain can be applied on the downlink side, namely transmission from the Base Station (BS) to User Equipment (UE) and the uplink, which is transmission from the UE to the BS.

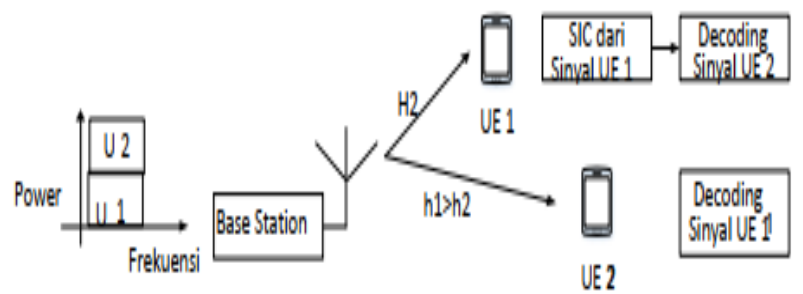

Figure 2. PD-NOMA scheme downlink transmission [10]

Figure 2 show an illustration of PD-NOMA with SIC on the downlink side. In the BS transmitter, the signal from each user is combined with the reference total power transmission throughput fairness of each user. In the receiver, SIC is used for multiuser detection (MUD). The near-far-effect condition causes the channel for each user to be different. Therefore, we need a SIC process that uses SINR to detect signals for each user.

\section{Successive Interference Cancellation (SIC)}

Successive interference cancelation (SIC) is one of the interference management methods. The SIC method is used in NOMA as multi-user detection on the receiver side [13]. Two or more signals from different users, allocated to the same subcarrier when transmitting, will certainly interfere with each other. By applying this SIC method to each receiver part, signals from other users who interfere will be selected so that the signal obtained will be good.

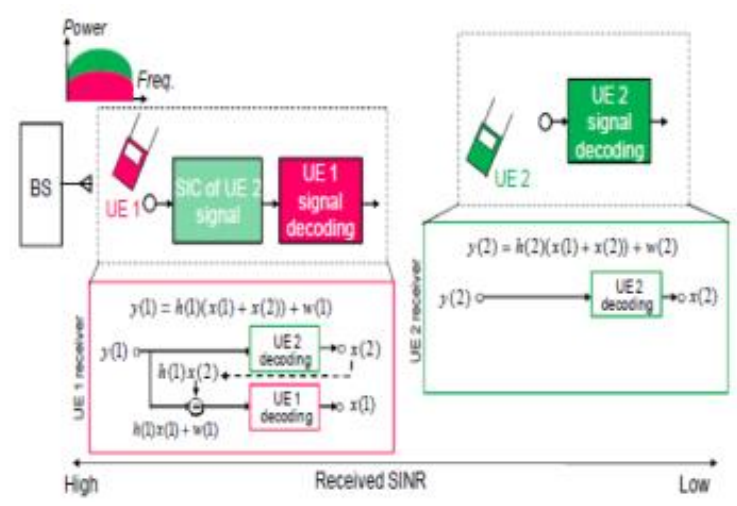

Figure 3. Successive Interference Cancellation Process [7]

Figure 3 show the process on the SIC on the receiver side. User Equipment 1 (UE1) the signal is detected with a greater power is $x_{1}$. The SIC process, the receive signal (y) subtracted from the detected signal $\left(x_{1}\right)$ so that the $x_{1}$ signal has passed through the Rayleigh channel along with 
the Additive White Gaussian Noise (AWGN) noise obtained during the transmission process $\left(h_{1} x_{1}+\right.$ $\left.w_{1}\right)$, then SIC output signal goes through the decoding process on User Equipment 1 (UE1). In the decoding process on User Equipment 2 (UE2), a signal with a smaller power was detected, namely $x_{2}$ [11]

$$
\begin{aligned}
x_{s}=\sum_{l=1}^{m_{s}} \sqrt{P_{s}}\left(i_{s}(l)\right) d_{s}\left(i_{s}(l)\right) \\
\\
y_{s}\left(i_{s}(i)\right)=h_{s}\left(i_{s}(l)\right) x_{s}+w_{s}\left(i_{s}(l)\right)
\end{aligned}
$$

\section{Definition:}

$x_{s}=$ modulation symbol from user $i_{s}$

$P_{S}\left(i_{s}(i)\right)=$ power allocation transmits for user $i_{s}$.

$y_{s}\left(i_{s}(i)\right)=$ dimensionless receive signal vector $N_{r}$ from user $i_{s}$ for every subcarrier from sub-band $s$.

$h_{s}\left(i_{s}(l)\right)=$ dimension coefficient vector channel $N_{r}$ from user $i_{s}$ of sub-band s. Coefficient vector channel contain of shadowing loss, distance dependent loss, instantaneous fading coefficient.

$w_{s}\left(i_{s}(l)=\right.$ vector noise plus inter-cell interference dimension $N_{r}$ pada subband $s$.

Equation of received signal SIC is: $y=h_{i} x+w_{i}$, where $x=\sqrt{p_{1} x_{1}}+\sqrt{p_{2} x_{2}}$, furthermore will be separated between $x_{1}$ and $x_{2}$, with criteria $p_{1}<p_{2}$.

\section{System Model}

Power allocation transmit used is fix power allocation (0.2 dB: $0.8 \mathrm{~dB}$; $0.3 \mathrm{~dB}: 0.7 \mathrm{~dB} ; 0.4 \mathrm{~dB}: 0.6 \mathrm{~dB}$ and 0.45 $\mathrm{dB}: 0.55 \mathrm{~dB}$ ). NOMA scheme used is based on the power domain. Simulated transmit and receive antenna configurations using Multiple Input Multiple Output (MIMO). The MIMO used in this research is $2 \times 2$, assuming that on the transmitter part, the data is divided into two, then sent through the channel, then at the receiver, the data from two receiving antennas are combined.

Block diagram simulation divided into three parts, there are transceiver block, channel block and receiver block. The simulation is carried out using Matlab software. SIC will be used as a multi-user detection which will separate the $U_{1}$ and $U_{2}$ receive signals. The simulation will be carried out by varying the value of the power domain and the speed of the user on each channel. The system performance can be analyzed from the BER vs SNR graph from the simulation results. The following is an illustration of PD-NOMA.

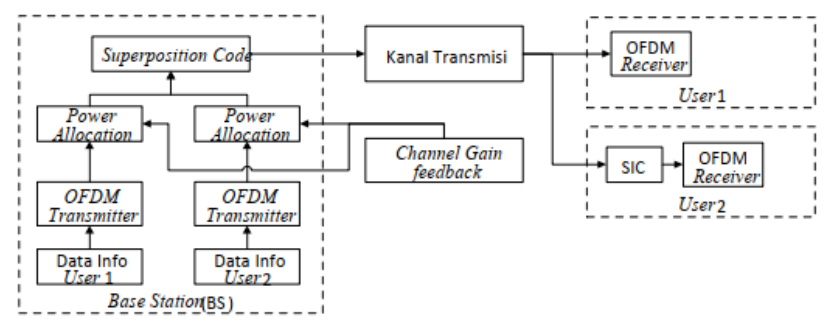

Figure 4. System block simulation of PD-NOMA
Figure 4 explains that at the base station (BS) information data for each user is processed in the OFDM block. The output signal from the OFDM block for each user is allocated a different power. The allocation of power is based on the feedback channel gain of each user. After the power of each user is allocated a different power, each user's signal is combined with a superposition code to be sent through the same resource.

At the user, signal received by the user with a low channel gain is immediately converted into information data with the receiver's OFDM block. While the signal received by the user with a high gain channel is processed by SIC to be separated from the user signal 1. After the desired signal has been successfully separated, the signal is returned to information data with the OFDM receiver block. The following is an explanation of each part of the transmitter, channel and receiver used in this study.

1. Transmitter block

Simulation parameter used in this research shown at Table 1.

Table 1. Simulation parameter

\begin{tabular}{ll}
\hline Information bit & 0 and 1 \\
\hline Number of data bit & 1.280 .000 \\
\hline Code rate & $1 / 2$ \\
\hline $\mathrm{K}$ & 7 dan 1 \\
\hline Point number FFT and IFFT & 128 \\
\hline
\end{tabular}

The functions used in the Matlab R2019a program in this research are shown in Table 2.

Table 2. Matlab Functions

\begin{tabular}{ll}
\hline \multicolumn{1}{c}{ Fungsi Matlab } & \multicolumn{1}{c}{ Keterangan } \\
\hline randi & Random data generator \\
\hline convenc & Convolution code \\
\hline polytrellis & Trellis structure \\
\hline ifft & IFFT process \\
\hline$f f t$ & FFT process \\
\hline
\end{tabular}

Figure 5 illustrate the block diagram that occurs at the transmitter. The process that occurs in this transmitter section is a data generator.

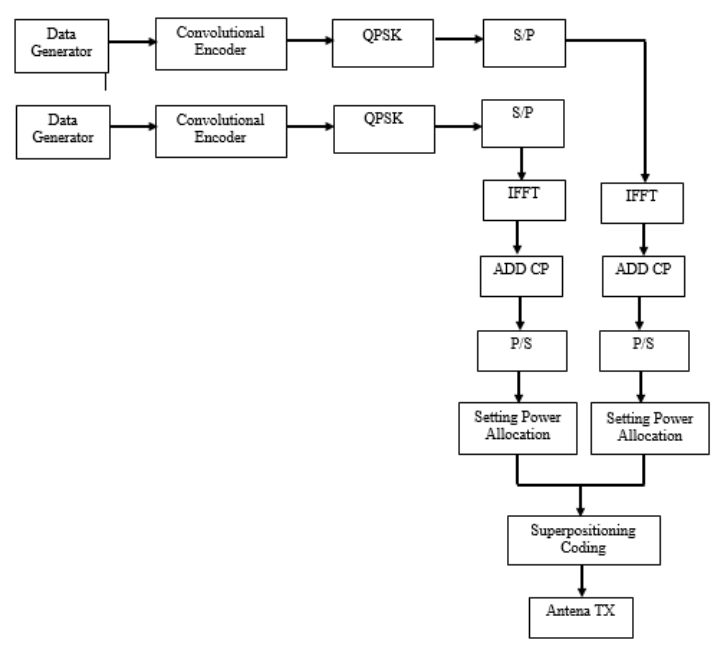

Figure 5. Transmitter block diagram 
Convolutional codes produce a codeword by passing bits of information through registers. The parameter to be used in the convolution is $C(j, K, l)$ where $j$ is the number of output bits, $K$ is the number of input bits, and $l$ is the amount of memory used.

Mapper or modulator is a block where the modulation process occurs. Modulation functions to map the bits of information into symbols before being superimposed on the carrier frequency. The modulation used in this simulation is QPSK. Inverse Fast Fourier Transfer (IFFT) block is a baseband modulator in OFDM system by generating subcarriers that are orthogonal between one subcarrier to another. Signals that have passed the IFFT process will be in the time domain.

ISI originating from the multipath channel effect can be reduced by using guard intervals. The cyclic prefix takes several samples of OFDM symbols as guard intervals to separate one symbol from another. In order to completely eliminate the effect of ISI, the length of the guard interval that must be allocated must be greater than the delay spread of the channel. So that the OFDM symbol block length equation becomes as follows:

$$
T_{s}=T_{g}+T_{b}
$$

Definition:

$T_{s}=$ OFDM period symbol after adding guard interval

$T_{g}=$ guard interval period

$T_{b}=$ symbol period before adding guard interval

Information data processed in OFDM is parallel data so it must be converted back into serial data for transmission.

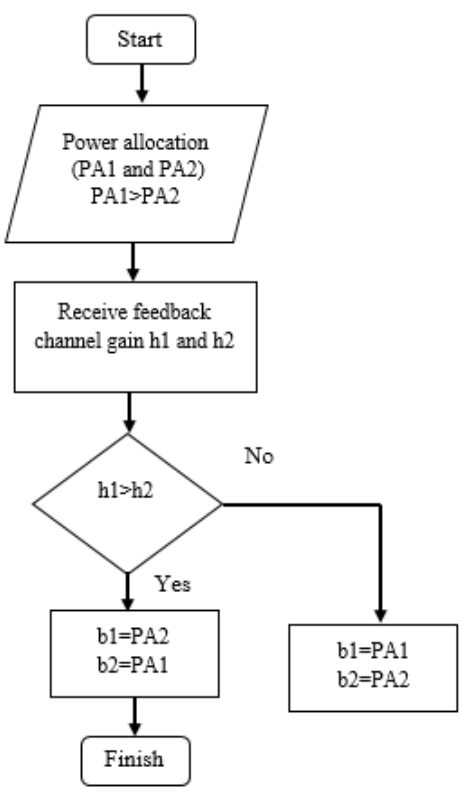

Figure 6. Power allocation user

Figure 6 illustrates the power allocation used depending on the channel gain value of each channel. Channels with high gain channels are allocated low send power, while for channels with low gain channels, high creasing power is allocated.

Superposition code is a technique of sending information simultaneously to multiple recipients through a single resource block. The superposition code can be recognized as a non-orthogonal scheme that achieves the capacity of a scalar gaussian broadcast channel. The equivalent of the signal transmitted by the BS with the superposition code for two users.

$$
x(n)=\sqrt{P \beta_{1}} S_{1}(n)+\sqrt{P \beta_{2}} S_{2}(n)
$$

Definition:

$P=$ total transmit power $($ Watt $)$

$\beta_{i}=$ fractions for allocation power $\left(\beta_{1}+\beta_{2}=1\right)$

\section{Transmission channel block}

Figure 7 describes the process that occurs in the transmission channel block used in this study. The transmission channel used in this simulation is noise with Adaptive White Gaussian Noise (AWGN). The signal received by the $i$-th user after passing through the channel with AWGN noise is as follows:

$$
y_{i}=h_{i} x+w_{i}
$$

Definition:

$h_{i}=$ channel gain of user- $i$

$w_{i}=\mathrm{AWGN}$ of user- $i$

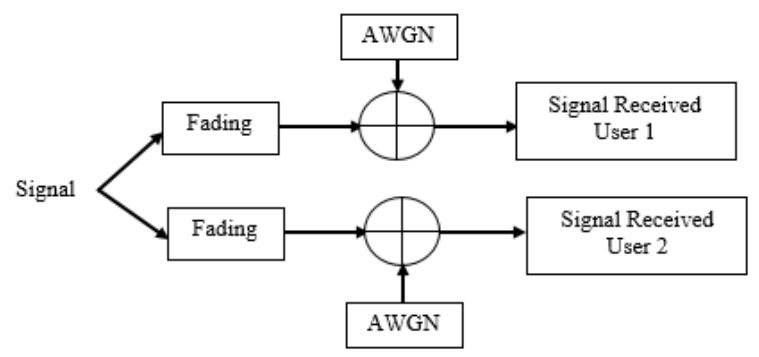

Figure 7. Transmission channel block

3. Receiver Block
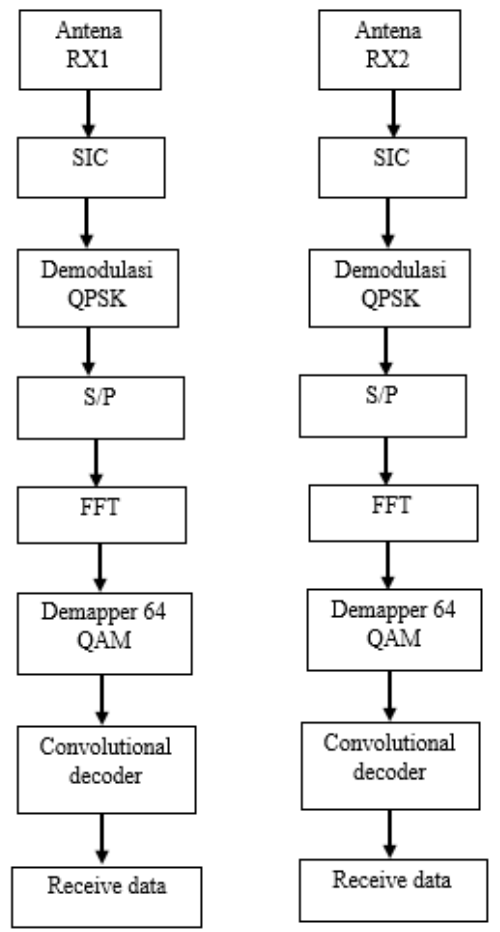

Figure 8. Receiver Block 
Figure 8 is the receiver part, where each receiver part, where each receiver part of the system is simulated. SIC is a process that will happen to each user on the receiver side. Figure 9 describes the flow of the SIC technique used in this study which is used to return code information to each information.

Removes the cyclic prefix that has been prepared previously on the sender block, so it will return to the OFDM symbol. In the serial to parallel part, it converts the series of bits received from the receiving antenna into a parallel series. Signal in the frequency domain that was previously in the time domain. In the demodulation process, the QPSK demodulation process is carried out, the symbols are mapped back into bits of information. The convolutional decoder process decodes the code word using Viterbi decoding to do the hard decision, which is to look for the bit value that is most similar to calculating the minimum hamming distance in a codeword. The value with the lowest hamming distance will also have a low bit rate error value.

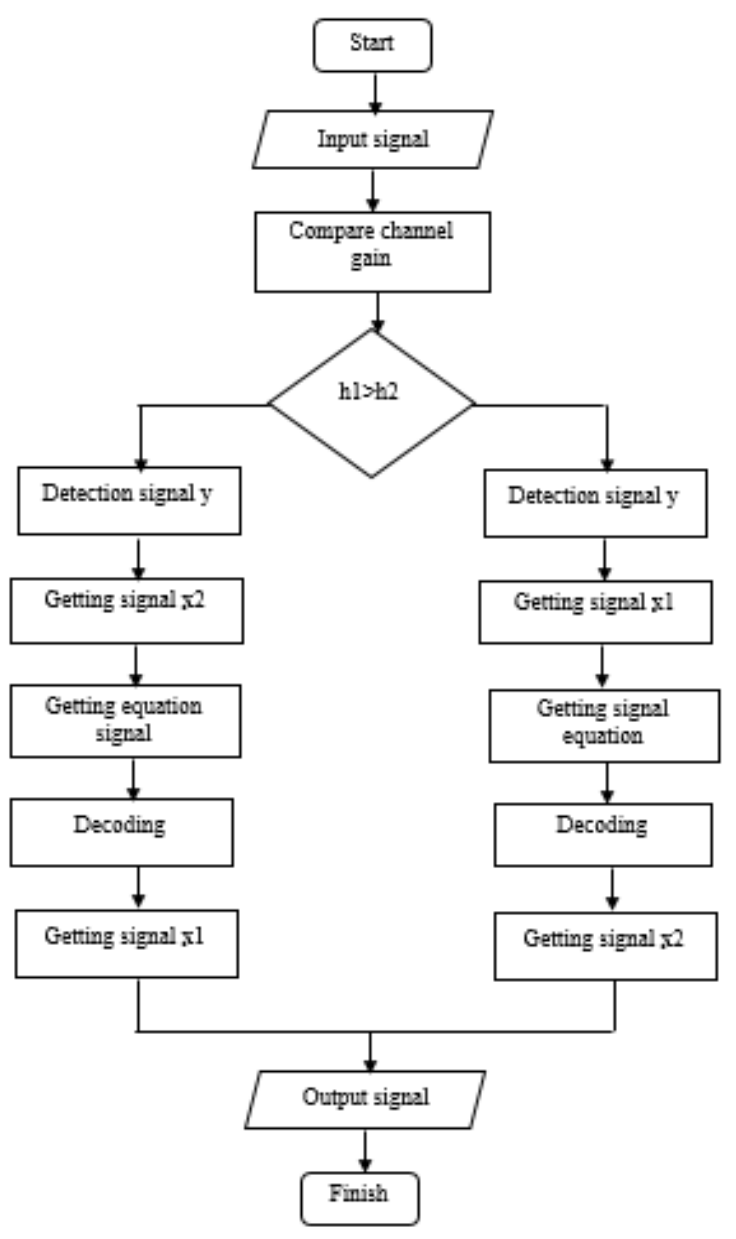

Figure 9. SIC flowchart

\section{System parameter}

Simulation has been carried out in accordance with the system modeling using Matlab R2019a. Simulation is carried out to see the system performance with BER parameter on SNR and channel capacity. This research uses the parameters listed in Table 3 .
Table 3. System Parameters

\begin{tabular}{lc}
\hline \multicolumn{1}{c}{ Parameters } & Value \\
\hline Frequency Carrier & $2 \mathrm{GHz}$ \\
\hline Total Subcarrier & 128 \\
\hline Types of Modulation & QPSK \\
\hline Power & $0,2 \mathrm{~dB} ; 0,8 \mathrm{~dB} ; 0,3 \mathrm{~dB} ;$ \\
& $0,7 \mathrm{~dB} ; 0.4 \mathrm{~dB} ; 0,6 \mathrm{~dB} ;$ \\
& $0,45 \mathrm{~dB} ; 0,55 \mathrm{~dB}$ \\
\hline \multirow{2}{*}{ Speed } & $40 \mathrm{~km} / \mathrm{hour}, 60 \mathrm{~km} / \mathrm{hour}$, \\
& $80 \mathrm{~km} / \mathrm{hour}$ and 100 \\
& $\mathrm{~km} / \mathrm{hour}$ \\
\hline Cycle Prefix & $1 / 4$ \\
\hline
\end{tabular}

\section{RESULT AND ANALYSIS}

1. Power Domain-NOMA analysis in SIC by power allocation ratio

Simulation process is carried out by dividing the power allocation of user 1 and user 2 to determine the performance of the BER and SNR values of the power provided by each user. In this simulation user 1 uses the SIC process and user 2 without carrying out the SIC process.

Figure 10 is the result of a simulation carried out using the power ratio between $U_{1}$ and $U_{2}$, which is $0.2 \mathrm{~dB}$ : $0.8 \mathrm{~dB}$. Based on the simulation results, $U_{1}$ has a channel gain value of 0.3533 and $U_{2}$ has a channel gain value of 0.2886 . This channel gain is the amplifier power of the channel. $0.2 \mathrm{~dB}$ power is allocated for $U_{1}$ (with SIC process) and $0.8 \mathrm{~dB}$ is allocated for $U_{2}$ (without SIC process) with the same BER value, BER $10^{-5}$ getting a greater SNR value at $U_{1}$ compared to $U_{2}$ with a difference of $7 \mathrm{~dB}$.



Figure 10. BER vs SNR with power allocation $0,2 \mathrm{~dB}: 0,8 \mathrm{~dB}$

The power comparison between $U_{1}$ and $U_{2}$ in Figure 11 is $0.3 \mathrm{~dB}: 0.7 \mathrm{~dB}$, for $U_{1}$ has a channel gain value of 0.3070 and $U_{2}$ has a channel gain value of 0.0454 . User 1 has a greater SNR value than user 2 .

Figure 12 simulation results carried out using the power ratio between $U_{1}$ and $U_{2}$ is $0.4 \mathrm{~dB}$ : $0.6 \mathrm{~dB}$. $U_{1}$ has a channel gain value of 0.1277 and $U_{2}$ has a channel gain value of 0.1759 , so for power allocation of $0.4 \mathrm{~dB}$ it is 
allocated to $U_{2}$ (with the SIC process). The simulation results show that the SNR value for $U_{2}$ is greater than that of $U_{1}$.

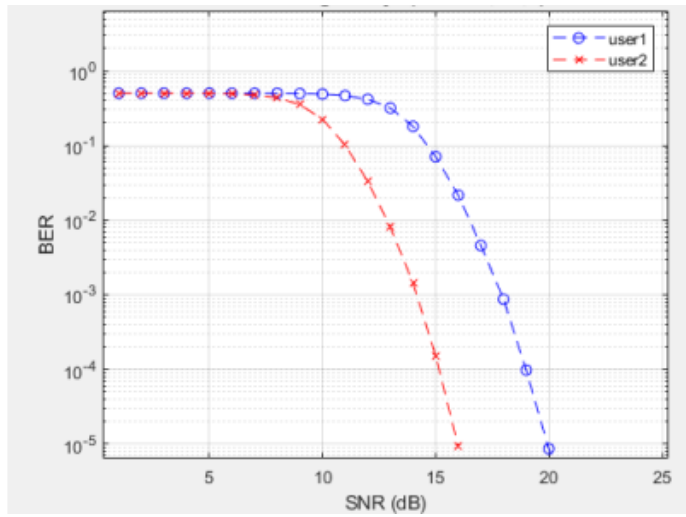

Figure 11. BER vs SNR with power allocation $0,3 \mathrm{~dB}: 0,7 \mathrm{~dB}$

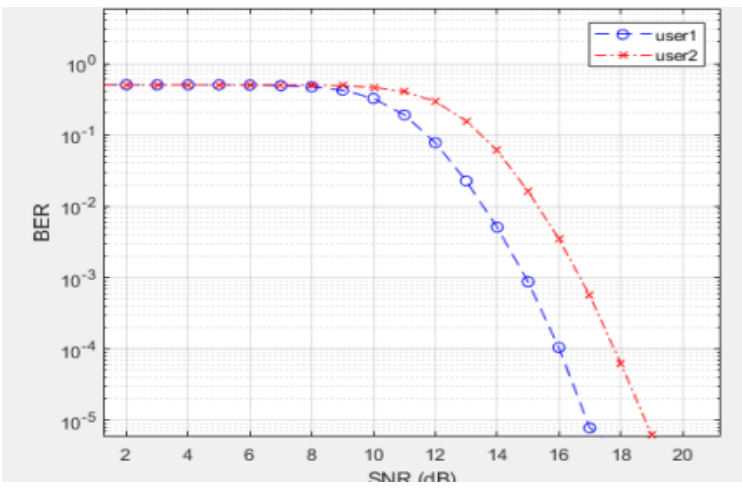

Figure 12. BER vs SNR with pwer allocation $0,4 \mathrm{~dB}: 0,6 \mathrm{~dB}$

Figure 13 is the simulation result performed using the power ratio between $U_{1}$ and $U_{2}$ is $0.45 \mathrm{~dB}: 0.55 \mathrm{~dB}$. Based on the simulation results, $U_{1}$ has a channel gain value of 0.1199 and $U_{2}$ has a channel gain value of 0.0872 .

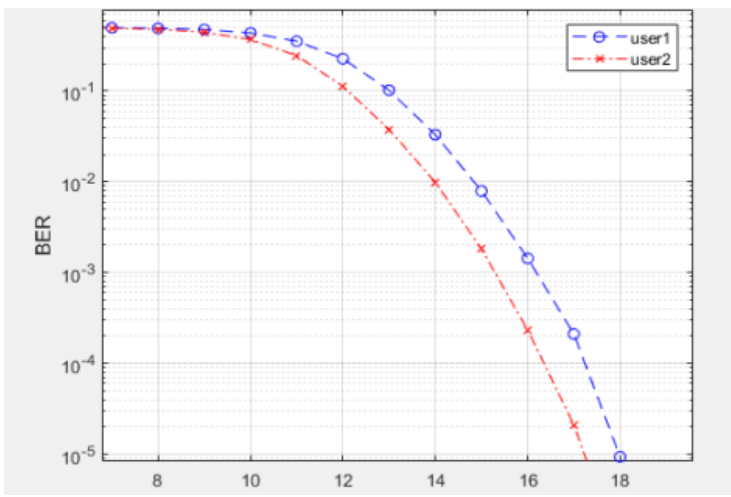

Figure 13. BER vs SNR with power allocation $0,44 \mathrm{~dB}: 0,45 \mathrm{~dB}$

Based on the simulation results of the power allocation comparison for each user, it is found that the power ratio of $0.45 \mathrm{~dB}$ : $0.55 \mathrm{~dB}$ produces the best BER vs SNR among other comparisons, because $U_{1}$ and $U_{2}$ get almost the same amount of power. So that to analyze the effect of variations in user mobility, the ratio of the sending power to be used is the ratio of the sending power of $0.45 \mathrm{~dB}$ : $0.55 \mathrm{~dB}$.
Based on the simulation results of the power allocation comparison for each user, it is found that the power ratio of $0.45 \mathrm{~dB}: 0.55 \mathrm{~dB}$ produces the best BER vs SNR among other comparisons, because user 1 and user 2 get almost the same amount of power. So that to analyze the effect of variations in user mobility, the ratio of the sending power to be used is the ratio of the sending power of $0.45 \mathrm{~dB}$ : $0.55 \mathrm{~dB}$.

Simulation to show the system performance with BER and SNR parameters. Table 4 is the simulation result of BER and SNR from power domain-NOMA for user 1 and user 2.

Table 4. Simulation result Power Domain NOMA BER $10^{-5}$

\begin{tabular}{lc}
\hline $\begin{array}{c}\text { Power allocation } \\
U_{1} \text { and } U_{2}\end{array}$ & $\begin{array}{c}\text { Signal to Noise Ratio (SNR) } U_{1} \\
\text { and } U_{2}\end{array}$ \\
\hline $0,2 \mathrm{~dB}: 0,8 \mathrm{~dB}$ & $22 \mathrm{~dB}: 15 \mathrm{~dB}$ \\
\hline $0,3 \mathrm{~dB}: 0,7 \mathrm{~dB}$ & $19 \mathrm{~dB}: 16 \mathrm{~dB}$ \\
\hline $0,4 \mathrm{~dB}: 0,6 \mathrm{~dB}$ & $17 \mathrm{~dB}: 19 \mathrm{~dB}$ \\
\hline $0,45 \mathrm{~dB}: 0,55 \mathrm{~dB}$ & $18 \mathrm{~dB}: 17 \mathrm{~dB}$ \\
\hline
\end{tabular}

2. PD-NOMS analysis on SIC with variations in user mobility

System testing is carried out with several different user speed conditions. The movement speed of user 1 is consistent, namely $40 \mathrm{~km} /$ hour. The first condition, the speed of $U_{1}$ and $U_{2}$ is set with a speed of $40 \mathrm{~km} /$ hour: $40 \mathrm{~km} /$ hour. The second condition is that the $U_{1}$ speed uses a speed of $40 \mathrm{~km} /$ hour and $U_{2}$ uses a speed of 60 $\mathrm{km} /$ hour. In the third condition $U_{1}$ uses a user speed of $40 \mathrm{~km} /$ hour, and $U_{2}$ uses a speed of $80 \mathrm{~km} /$ hour. In the last state, $U_{1}$ uses a speed of $40 \mathrm{~km} /$ hour and $U_{2}$ uses a speed of $100 \mathrm{~km} /$ hour. The following is the simulation result on network performance in the form of BER vs SNR.

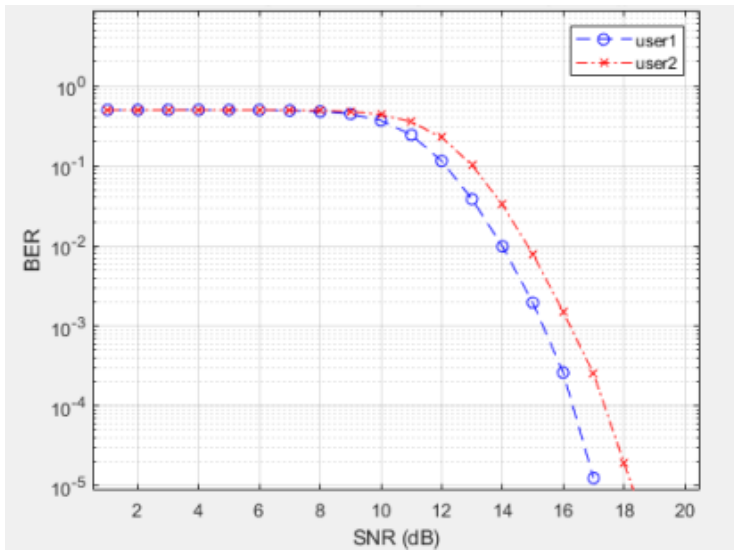

Figure 14. BER vs SNR with $V_{1}, V_{2}=40 \mathrm{~km} / \mathrm{jam}$

Figure 14 shows the BER vs SNR performance obtained from the NOMA system with the condition that the user is moving at the same speed, $U_{1} 40 \mathrm{~km} /$ hour and $U_{2} 40 \mathrm{~km} /$ hour. The SNR value obtained by $U_{1}$ is smaller when compared to $U_{1}$. 


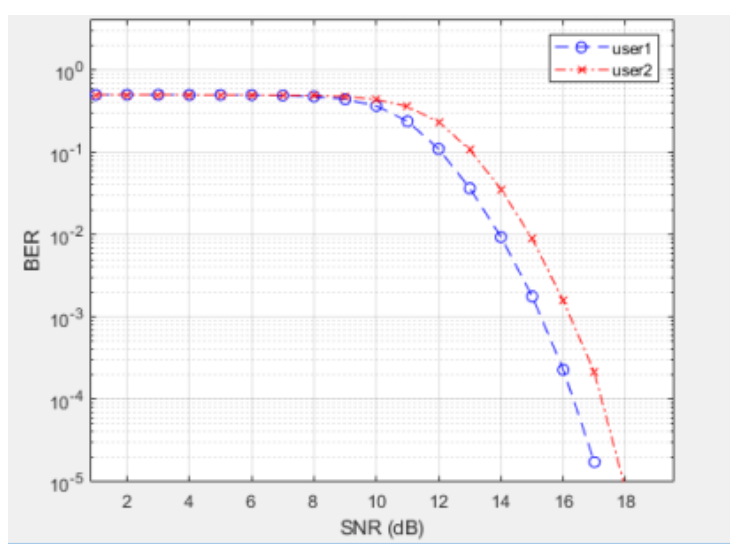

Figure 15. BER vs SNR with $V_{1}: V_{2}=40: 60 \mathrm{~km} / \mathrm{jam}$

Figure 15 shows the BER vs SNR performance of the NOMA system with the condition that the user is moving at a speed of $U_{1} 40 \mathrm{~km} /$ hour and $U_{2} 60 \mathrm{~km} /$ hour. The SNR value obtained by $U_{1}$ is still smaller when compared to $U_{2}$ even though the speed between $U_{1}$ and $U_{2}$ is different by $20 \mathrm{~km} /$ hour.

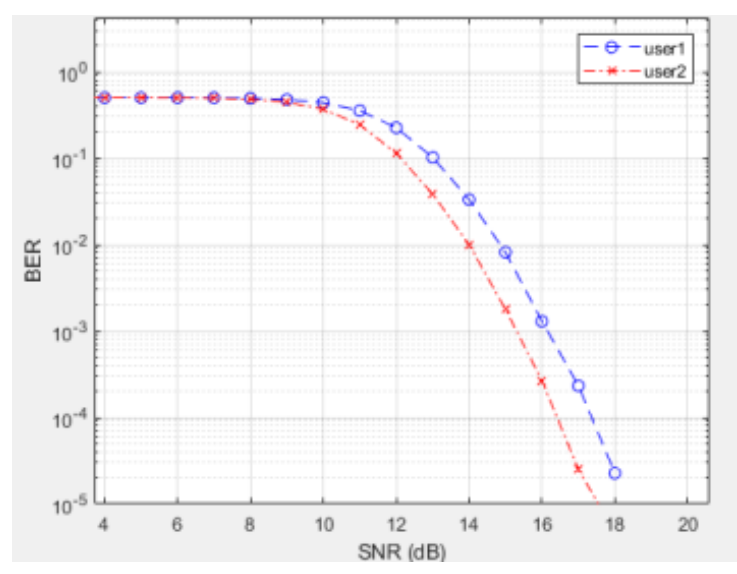

Figure 16. BER vs SNR with $V_{1}: V_{2}=40: 80 \mathrm{~km} / \mathrm{jam}$

Figure 16 shows the BER vs SNR performance obtained from the NOMA system with the user moving at the same speed, namely $U_{1} 40 \mathrm{~km} /$ hour and $U_{2} 80 \mathrm{~km} /$ hour. The SNR value obtained by $U_{1}$ is greater than that of $U_{2}$ even though the speed between $U_{1}$ and $U_{2}$ is differentiated by $40 \mathrm{~km} /$ hour.

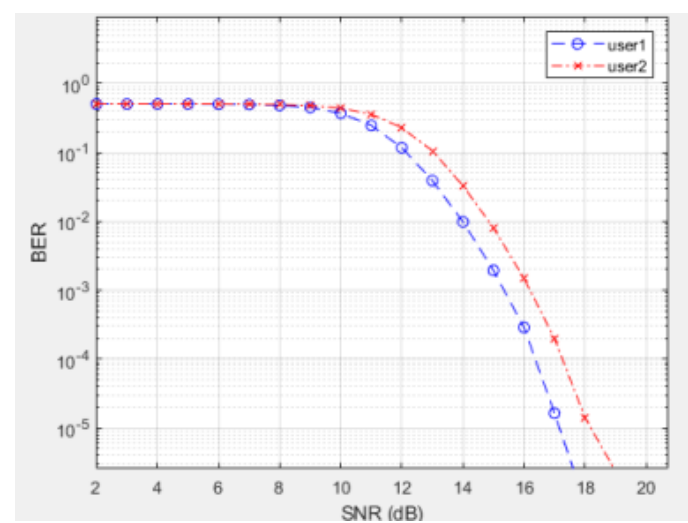

Figure 17. BER vs SNR with $V_{1}: V_{2}=40: 100 \mathrm{~km} / \mathrm{jam}$

Figure 17 shows the BER vs SNR performance obtained from the NOMA system with the condition the user is moving at a speed of $U_{1} 40 \mathrm{~km} /$ hour and $U_{2} 100 \mathrm{~km}$ / hour. BER $10^{-5}$ produces a different SNR between the two users where $U_{1}$ has a smaller SNR value when compared to $U_{2}$ even though the speed between $U_{1}$ and $U_{2}$ is different by $60 \mathrm{~km} /$ hour.

Table 5. Simulation result PD-NOMA of mobility user BER $10^{-5}$

\begin{tabular}{cc}
\hline $\begin{array}{c}\text { Variation mobility user } \\
U_{1} \text { and } U_{2}\end{array}$ & $\begin{array}{c}\text { Signal to Noise Ratio (SNR) } \\
U_{1} \text { dan } U_{2}\end{array}$ \\
\hline $40 \mathrm{~km} /$ hour: $40 \mathrm{~km} /$ hour & $17 \mathrm{~dB}: 18,1 \mathrm{~dB}$ \\
\hline $40 \mathrm{~km} /$ hour: $60 \mathrm{~km} /$ hour & $17,3 \mathrm{~dB}: 18 \mathrm{~dB}$ \\
\hline $40 \mathrm{~km} /$ hour: $80 \mathrm{~km} /$ hour & $18 \mathrm{~dB}: 17,8 \mathrm{~dB}$ \\
\hline $40 \mathrm{~km} /$ hour: $100 \mathrm{~km} /$ hour & $17,9 \mathrm{~dB}: 19 \mathrm{~dB}$ \\
\hline
\end{tabular}

The graph of the simulation results to observe the effect of speed variations of each user, the greater the difference in speed between $U_{1}$ and $U_{2}$, the gap between the BER vs SNR performance for both users is getting smaller. This occurs because of the power allocation process before the signal transmission process, in line with the power allocation principle, channels with good quality will be allocated low sending power compared to channels that have poor channel quality.

Users with faster movements will certainly be allocated higher power compared to users who move slower. So that both users get almost the same signal quality. It's different if the user's speed is the same or the difference is small. Because the quality of the two channels is almost the same while the allocation of the sending power is different, the quality of the received signal between the two users will of course also be different, depending on the allocation of the sending power of each user.

\section{CONCLUSION}

This research has simulated the effect of power allocation and user mobility using SIC. The result obtained from the effect of power allocation is that the greater the difference in power allocation sent between $U_{1}$ and $U_{2}$ will result in a performance value that has a bigger gap, and vice versa. BER vs SNR is best when using the ratio of $0.45 \mathrm{~dB}: 0.55 \mathrm{~dB}$ of transmittable power, because $U_{1}$ and $U_{2}$ get almost the same amount of power at $U_{1}$, the BER value $10^{-5}$ when SNR is $18 \mathrm{~dB}$ and on $U_{2}$ SNR is $17 \mathrm{~dB}$. User mobility performance with speed $U_{1}$ and $U_{2}=$ $40 \mathrm{~km} /$ hour get the best SNR value, which is $18 \mathrm{~dB}$ at BER $\nabla 10^{-5}$. The results showed that the power allocation of the sending power greatly affects the power received by each user and the results of the mobility of each user do not significantly affect the performance received by each user. For further system testing, you can use channel estimation and apply adaptive modulation.

\section{REFERENCES}

[1] Yuya Saito, Anass Benjebbour, Yoshihisa Kishiyama, Takehiro Nakamura, "System-level performance of Downlnk Non-Orthogonal Multiple Access (NOMA) under Various Environments, 5G Radio Access Network Research Group 5G Laboratory" NTT DOCOMO, INC, 2012. 
[2] IMT-2020, "5G Concept," ournal of Chemical Information and Modeling, vol. 53, no. 9, pp. 1689-1699, 2013.

[3] M. Riazul Islam, "Power-Domain Non-Orthogonal Multiple Access (NOMA) in 5G System : Potentials and challenges," IEEE Commun. Surv. Tutorials, vol. 19, no. 2, pp. 721-742, 2017.

[4] W. Bao, "Joint Rate Control and Power Allocation for Non-orthogonal Multiple Access System," IEEE J. Sel. Areas Commun, vol. 35, no. 12, pp. 2798-2811, 2017.

[5] L.P Qian, "Resource Management in Non-Orthogonal Multiple Access Network for 5G and Beyond," no. August, pp. 15-21, 2017.

[6] Y.Saito,Y.Kishiyama,A.Benjebbour, T. Nakamura, A. Li, Kenichi Higuchi "Non-Orthogonal Multiple Access (NOMA) for CellularFutue Radio Access" DOCOMO Beijing Communications Laboratories Co.,Ltd, 2013

[7] Dian Ratna Kumala "Analisis Performansi Akses Jamak Non Orthogonal Multiple Access (NOMA) Dengan Successive Interference Cancellation (SIC) Untuk Teknologi Seluler Masa Depan," Universitas Telkom, Bandung, 2016.

[8] L. Dai, "Non-Orthogonal multiple access for 5G : Solutions, challenges, opportunities, and future research trends," IEEE Commun. Mag, vol. 53, no. 9, pp. 74-81, 2015.

[9] Annas Benjebbour, Anxin Li, Yuya Saito, Yoshihisa Kishiyama, Atsushi Harada, Takehiro Nakamura, "System-Level Performance of Downlink NOMA for Future LTE Enchacements," Radio Access Network Development Departemen, NTT DOCOMO, INC DOCOMO, Beijing Communications Laboratories Co,.Ltd, 2013

[10] Uke. Usman, Fundamental Teknologi Selular Long Term Evolution (LTE), Bandung: Rekayasa Sains, 2012.

[11] K. Abdillah, Y.Moegiharto, "Analisa Kinerja Orthogonal Frequency Division Multiplexing (OFDM) Berbasis Perangkat Lunak," Politeknik Elektro Negeri SurabayaInstitut Teknologi Sepuluh November (PENS-ITS), Surabaya, 2010

[12] O.Maraqa, Aditya S, dkk, "A Survey of Rate-optimal Power Domain NOMA with Enabling Technologies of Future Networks". IEEE Communication Vol.20, No.20, July 2020.

[13] B.W.Dai, "Non-Orthogonal Multiple Access for 5G", IEEE Communication, vol.53., no.9, pp.74-81, 2015. 